\title{
FATORES INFLUENCIADORES NA IMPLANTAÇÃO EM LARGA ESCALA DOS TABLETS EDUCACIONAIS NO BRASIL
}

\author{
Giovanni Ferreira de Farias, Ed.D. - EGC/UFSC - giovanni.farias@gfarias.com \\ Fernando José Spanhol, Dr. - EGC/UFSC - fernando.spanhol@ufsc.br
}

\begin{abstract}
Resumo
Várias iniciativas de implantação de tablets educacionais em larga escala aconteceram nos últimos dez anos, e junto vieram muitos problemas de implantação, levando inclusive à suspensão ou cancelamento das iniciativas. No Brasil, onde foi anunciada a implantação de 600.000 tablets educacionais, vários estudos de caso trataram do assunto, porém enfocando principalmente o contexto da sala de aula. A pesquisa aqui relatada, por outro lado, enfoca especificamente a implantação em larga escala dos tablets educacionais no Brasil. Usando em Grounded Theory, esta pesquisa identificou dezesseis fatores preponderantes neste tipo de implantação a partir de relatos de quem acompanhou o processo em dezenas de escolas de diferentes regiões do país. O resultado foi o modelo de implantação MILETE (Modelo de Implantação em Larga Escala de Tablets Educacionais), o qual pode nortear gestores educacionais em processos de implantação semelhantes.
\end{abstract}

Palavras-chave: implantação, larga escala, tablet educacional, escolas públicas, Brasil.

\section{INFLUENCING FACTORS FOR THE LARGE-SCALE DEPLOYMENT OF EDUCATIONAL TABLET COMPUTERS IN BRAZIL}

\begin{abstract}
Several initiatives of large-scale deployment of educational tablet computers have been occurred in the last ten years, along with many implementation problems, including suspension and canceling. In Brazil, where it was announced the deployment of 600,000 educational tablet computers, several case studies addressed this topic, but focusing mostly the classroom context. The research herein reported, on the other hand, focuses specifically the large-scale deployment of the educational tablet computers in Brazil. Using Grounded Theory, this research identified sixteen preponderant factors in this kind of implementation from the testimonies of who followed up the process in dozens of schools from different regions of the country. The result was the implementation model LSMDD (Large Scale Mobile Device Deployment, which can guide educational managers in similar deployment processes. and documents related to the mobile device in dozens of educational institutions. Key words: deployment, large scale, educational tablet computer, public school, Brazil.

\section{Introdução}

A percepção da necessidade de aprimoramento da educação pública, em especial do ensino médio, no Brasil se deu principalmente pelos indicadores educacionais nacionais, como IDEB (Índice de Desenvolvimento da Educação Básica) (Terra, 2012), e internacionais, como PISA (Programme for International Student Assessment), promovido entre as nações da OCDE (Organização para Cooperação e Desenvolvimento Econômico) (OCDE, 2012). Em ambos indicadores, o nível detectado da educação básica no Brasil foi considerado bastante baixo em relação às expectativas relativas a um país com o nível de desenvolvimento como o brasileiro. Na avaliação do PISA, por exemplo, o Brasil ficou em
\end{abstract}


$55^{\circ}$ lugar numa lista de 65 países (OCDE, 2012). A avaliação do IDEB em 2011 também não apresentou boas perspectivas para o ensino brasileiro, especialmente o público, que puxou para baixo a média de avaliação do ensino médio com uma média de 3,4, resultando numa média total de 3,7 graças à ajuda da média do ensino privado, que alcançou 5,4 (INEP, 2016)

Mediante esta situação, o governo Brasileiro anunciou em 2012 o lançamento de um projeto ambicioso para melhorar a qualidade do ensino básico no Brasil. O objetivo inicial era implantar 600.000 tablets educacionais em escolas públicas dedicadas a este nível de educação ao longo de todo o território nacional. O projeto também incluía a distribuição de 3.700 lousas digitais para que, junto com os tablets educacionais, os professores pudessem aprimorar as suas respectivas abordagens em sala de aula, com apresentações de conteúdo mais dinâmicas e atraentes, contando com objetos de aprendizagem como textos, imagens, áudios, animações e vídeos. Desta forma, o resultado esperado seria a conquista de uma melhor atenção do aluno às aulas dadas, a disponibilização de recursos mais ricos pedagogicamente e o consequente alcance de melhores resultados de aprendizagem (Santana; Rossine; Pretto, 2012; Mercadante, 2013; Lima, 2013).

Portanto, o lançamento do projeto de implantação dos tablets educacionais foi anunciado como sendo um importante passo do governo federal no sentido de aprimorar o processo de ensino aprendizagem brasileiro no seu sistema educacional público. Apesar disso, a ideia não foi bem aceita por parte dos educadores. Cardoso (2012), por exemplo, declarou que o entusiasmo do governo no início da implantação levaria a pouco ou nenhum resultado significante a partir do uso da tecnologia envolvida. Ela foi categórica ao afirmar que tablets educacionais é "mais do mesmo", ou seja, é mais um caso de implantação de tecnologia da educação com resultados pífios. Ela fez coro com as palavras de Jens Bammel, que comentou sobre a onda de implantação de tablets educacionais, durante o $3^{\circ}$ Congresso Internacional do Livro Digital, em São Paulo, afirmando que "em todo o mundo, surgem políticos dizendo que é preciso trazer a sala de aula para o século XXI enquanto tiram fotos com iPads ao lado de crianças sorridentes" (Cardoso, 2012).

Teles (2012) reforçou as críticas ao projeto de implantação dos tablets educacionais ao destacar a falta de um currículo com planejamento mais amplo para suportar o uso dos tablets educacionais, bem como a inexistência de padrões para o conteúdo digital a ser usado pelos professores através de tais dispositivos móveis. Ferreira (2013) foi ainda mais pragmático em suas críticas ao lembrar que muitas escolas nem tinham a adequada conectividade com a Internet, nem contavam com professores capacitados para lidar com tais dispositivos. Aliás, até a capacitação anunciada pelos defensores da implantação dos tablets foi criticada por conta de sua brevidade e superficialidade (Lima, 2013).

Apesar da importância do tema e do impacto operacional e financeiro para gestores educacionais ao redor do mundo, segundo McGee e VanderNoor (2013), pouco se estuda sobre os fatores que influenciam o sucesso ou o fracasso destas operações de implantação de tecnologia educacional em larga escala. Há diversos aspectos a serem considerados em uma implantação deste tipo, tais como infraestrutura, capacitação, logística, manutenção, regulação entre outros. A implantação dos tablets respaldou estas críticas, pois a recusa dos professores em usar os dispositivos, problemas com hardware, baixa adesão às ações de capacitação no uso do tablet que ocorrem via Internet e a falta de conectividade adequada para fazer uso efetivo do equipamento em sala de aula (Neves \& Cardoso, 2013). Vale salientar que tais problemas também são encontrados em iniciativas semelhantes no 
exterior, como mostra a Tabela 1, considerando um estudo que levantou implantações envolvendo mais de 100.000 dispositivos (Farias e Ally, 2015).

Tabela 1 - Principais implantações de tablets educacionais em larga escala no exterior.

\begin{tabular}{|c|c|c|c|}
\hline País & $\begin{array}{l}\text { Número de } \\
\text { dispositivos }\end{array}$ & $\begin{array}{c}\text { Ano de } \\
\text { lançamento }\end{array}$ & Fatos que marcaram a implantação \\
\hline Cingapura & 120.000 & 2010 & $\begin{array}{l}\text { Sofreu dezoito meses de atraso na implantação, além de uma } \\
\text { série de imprevistos tecnológicos e logísticos (Kwang, 2012). }\end{array}$ \\
\hline Tailândia & 860.000 & 2012 & $\begin{array}{l}\text { A baixa qualidade do hardware empregado resultou numa taxa } \\
\text { de } 30 \% \text { de tablets defeituosos (Sakawee, 2013). }\end{array}$ \\
\hline Índia & 100.000 & 2013 & $\begin{array}{l}\text { O projeto seria cancelado pelo baixo desempenho do hardware, } \\
\text { mas foi trocado por outro melhor (Nanda e Agarwal, 2012). }\end{array}$ \\
\hline Turquia & 15.000 .000 & 2013 & $\begin{array}{l}\text { Enfrentou problemas com conectividade, compatibilidade de } \\
\text { tablets e projetores, e conteúdo. Implantou 5\% do planejado. } \\
\text { (Daily Sabah, 2014; Todasy's Zaman, 2015). }\end{array}$ \\
\hline Estados Unidos & 640.000 & 2013 & $\begin{array}{l}\text { Projeto foi suspenso por tempo indeterminado, devido à falta } \\
\text { de conteúdo e currículo adequados, uso inapropriado do } \\
\text { dispositivo e conectividade insuficiente (Kastrenakes, 2014). }\end{array}$ \\
\hline
\end{tabular}

Porém, uma busca sistemática realizada para este estudo constatou que há uma abundância de publicações focadas na aceitação de adoção da tecnologia dos tablets e metodologias de ensino-aprendizagem com o uso dos dispositivos móveis (Valstad 2011; 2011; Donaldson, 2011; Clarke \& Svanaes, 2012; Wang \& Huang, 2015), e os resultados apresentados são quase sempre bastante positivos em relação ao uso destes dispositivos no contexto educacional. Porém, confirmando os achados de McGee e VanderNoor (2013), praticamente nada se publica sobre aspectos operacionais da implantação de larga escala de tablets educacionais.

O principal elemento diferenciador das tentativas de implantação e os relatos de casos de uso de tablets em sala de aula é o processo de implantação em si, especialmente pela escala envolvida. Os relatos não fazem qualquer citação sobre o processo que precedeu o uso dos tablets que é descrito positivamente, e apenas descrevem processos que envolvem no máximo algumas poucas centenas de unidades (Tront, 2007; Sommerich et al, 2007; Bassi, 2010). Dentre publicações brasileiras sobre o tema, basicamente foram encontrados estudos voltados para as percepções dos professores sobre aspectos diretamente relacionados com os mesmos no processo de implantação (Lucas, 2014; Nascimento, 2014; Souza, 2014; Neves \& Cardoso, 2013; Moura, 2015; Oliveira, 2016) ou sobre algum aspecto específico da implantação, como a formação dos professores no uso dos tablets educacionais (Rodrigues, 2016; Quaresma et. al., 2014).

Este estudo, portanto, veio a preencher esta lacuna, suscitando o seguinte questionamento: se há tantos relatos positivos do uso de tablets em sala de aula, então quais fatores influenciam a implantação de tablets educacionais em larga escala para se chegar a tantos problemas de implantação?

\section{Material e Métodos}

Como o cenário de pesquisa foi o brasileiro e a implantação em larga escala em curso era no setor público, o objetivo primordial da pesquisa foi definir, de uma forma sistemática, os principais fatores a serem observados no processo de implantação de tablets educacionais em escolas públicas no Brasil. Além do mais, este estudo visou identificar 
eventuais relações entre tais fatores, de modo a compor um modelo de implantação que fornecesse diretrizes úteis para gestores educacionais em processos de implantação em larga escala de tablets educacionais.

Desta forma, fazendo uso da abordagem descritiva de pesquisa preconizada por Creswell (2009), este estudo pode ser definido como tendo base epistemologica intepretativista, faz uso de Teoria Fundamentada em Dados, mais frequentemente denominada de Grounded Theory (termo usado neste texto), como metodologia de pesquisa (Corbin e Strauss, 2008). Conta com métodos de pesquisa baseados em questionário online e entrevista semi-estruturados e observação in-loco no ambiente de implantação.

A escolha metodológica desta pesquisa foi justificada pela complexidade do cenário de estudo, com inúmeras variáveis a serem consideradas, além da dificuldade em se levantar preliminarmente hipóteses, por conta do fenômeno estudado ser muito recente e pela falta de estudos prévios mais diretamente relacionados com o objetivo da pesquisa.

Assim, os dados que alimentam a pesquisa se originam a partir do fato de que seu resultado se baseia nas percepções dos envolvidos na implantação dos tablets educacionais no Brasil, expressas em forma de respostas a questões abertas, sejam realizadas via formulário online ou via entrevista.

Como pode ser verificado na Figura 1, o processo de pesquisa começou pela coleta inicial dos dados, feita com o suporte da ABED (Associação Brasileira de Educação a Distância), que usou sua base de emails para que convites para participação na pesquisa fosse feito àqueles que tiveram alguma relação com o processo de implantação dos tablets.

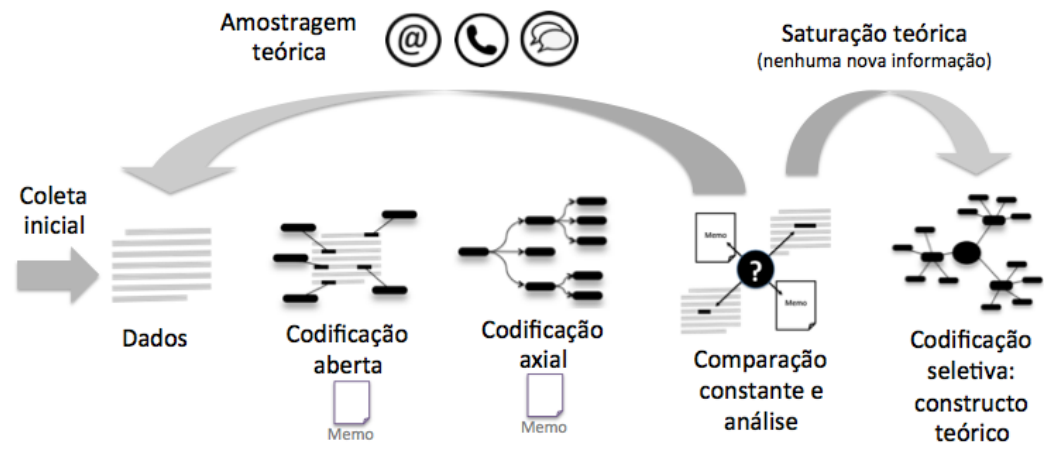

Figura 1 - Processo de pesquisa baseado em Grounded Theory.

Os dados iniciais foram coletados a partir de formulário online, com campos referentes a dados de perfil do participante e questões sobre o processo de implantação. Os dados foram analisados e foi feita uma codificação aberta, que implica em se chegar a conceitos importantes contidos nos depoimentos dos participantes. Em seguida, foi feita a codificação axial, que implica em aglutinar códigos relacionados a conceitos de mesma natureza, gerando categorias de conceitos, as quais já eram os fatores perseguidos nesta pesquisa. Ao mesmo tempo em que tais codificações eram feitas, eram criadas anotações, chamados neste texto de memos, com observações e conclusões chegadas pelo pesquisador sobre as análises realizadas.

Então, foi feita uma análise de comparação constante e análise dos códigos, categorias e memos para identificar possíveis lacunas ou inconsistências nos dados analisados, bem como novos conceitos e informações que eventualmente passaram despercebidos nas codificações. Uma vez que se identificou lacunas conceituais ou necessidade de mais investigação, foi feita uma amostragem teórica, que implicou em 
realizar nova coleta de dados, seja com os participantes antigos, seja com novos participantes, tomando como base o tipo de dado que se buscava.

Através de contatos pessoais, via email ou por telefone, visitas foram feitas a escolas e nova coleta de dados foi realizada, para reiniciar o processo de codificação aberta e axial. Este processo iterativo seguiu até não haver mais novas informações surgidas da análise e comparação constante dos dados. A saturação teórica foi atingida, portanto. Passou-se assim a fazer a codificação seletiva, que consistiu na identificação da principal categoria, ou fator influenciador na implantação em larga escala dos tablets educacionais, e o estabelecimento das relações entre ela e as outras categorias. Como resultado, se chegou a um constructo teórico que podemos chamar de modelo de implantação em larga escala de tablets educacionais.

\section{Resultados e Discussão}

A pesquisa foi executada no último trimestre de 2015, já totalizando três anos após o início do processo de implantação dos tablets. Desta forma, houve tempo suficiente para que os participantes envolvidos tivessem opinião formada sobre o processo de implantação, bem como conhecimento sobre os fenômenos que o cercam. O formulário online coletou 82 respostas da primeira leva de participantes, das quais 17 foram excluídas por serem incompletas ou os respondentes não atenderem ao perfil exigido para sua participação. Restaram, portanto, 65 respostas vindas de todas as regiões do Brasil.

A primeira informação analisada foi o perfil dos participantes, composta de dados sobre idade, gênero, unidade da federação, nível educacional, experiência profissional, tipo de envolvimento na implantação do tablet educacional e, para os que declararam serem professores, a disciplina que ensinam ou ensinavam nas escolas envolvidas no uso dos dispositivos móveis. Esta análise demonstrou que o perfil dos participantes não indicou ser fonte geradora de viés de pesquisa que pudesse ser considerada comprometedora para as respostas das questões abertas.

Após a primeira codificação aberta, chegou-se a cinquenta códigos, e com a codificação axial, foram identificados trinta e três categorias iniciais. Feita a amostragem teórica inicial, iniciou-se um processo de visitação à escolas ao longo do país, o que propiciou a inclusão de quinze novos participantes para amostragem teórica nos ciclos de Grounded Theory subsequentes. Estes participantes foram entrevistados pessoalmente, estando os mesmos distribuídos em escolas localizadas em cinco cidades: Campina Grande/PB, Vila Velha/ES, Rio de Janeiro/RJ, Criciúma/SC e Araranguá/SC. Estas visitas também permitiram que o pesquisador observasse in loco alguns fenômenos descritos pelos respondentes de todo o país. Tais observações geraram registros em memos, e foi um importante instrumento para implementação da pesquisa baseada em Grounded Theory.

Ao alcançar a saturação teórica, se chegou a dezesseis categorias, correspondentes aos fatores influenciadores buscados pelo estudo, mostradas com suas respectivas definições na Tabela 2. A relação entre os fatores encontrados resultou no MILETE (Modelo de Implantação em Larga Escala de Tablets Educacionais), mostrado na Figura 2, o qual pode nortear gestores educacionais em processos de implantação semelhantes.

Um fato lamentável é que a expectativa inicial era que os fatores aqui encontrados fossem baseados na descrição de práticas tanto recomendáveis como não recomendáveis, trazendo experiências positivas e negativas. Porém, o que se percebeu é que esta pesquisa 
se baseou majoritariamente em experiências negativas referentes ao processo de implantação dos tablets, que atestavam más práticas e frustrações.

Os depoimentos coletados e as observações feitas durantes as visitas destacam alguns fatos muito representativos de como tem ocorrido o processo de implantação dos tablets educacionais no Brasil. Foram encontradas escolas sem qualquer conectividade em sala de aula, ao mesmo tempo que os dispositivos que não já estava quebrados tinham pouca memória, o que impedia que o professor baixasse conteúdo na sala dos professores para posteriormente apresentar em sala de aula, como uma forma de contornar a falta de conectividade. Numa escola em específico, dos 20 tablets recebidos, apenas um estava em uso, sendo o restante rejeitado pelos professores ou devolvidos por aqueles que ainda tiveram o ímpeto de se engajar no projeto. A reclamação que o tablet representava um aumento de carga de trabalho e de responsabilidade, desprovido de capacitação adequada era generalizada em todas as escolas visitadas.

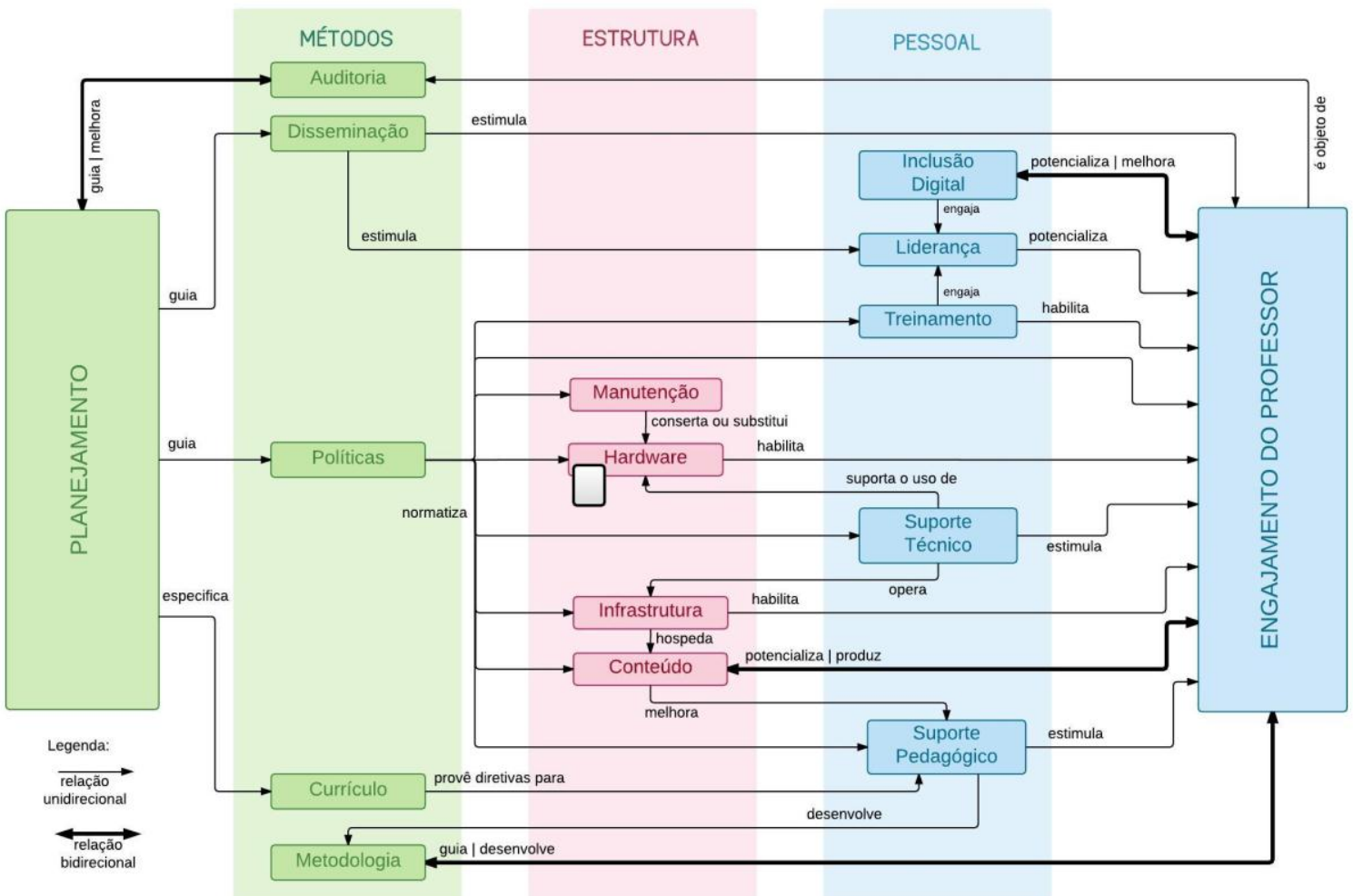

Figura 2 - MILETE (Modelo de Implantação em Larga Escala de Tablets Educacionais).

Houve também muitas queixas com a qualidade do dispositivo, que quando apresentava algum defeito passava meses na manutenção, sem se ter dispositivos substitutos. Em algumas escolas, a garantia de manutenção já tinha vencido quando os tablets efetivamente começaram a ser usados.

O responsável pelo suporte técnico de uma escola chegou a afirmar que o seu diretor não tinha interesse que o projeto desse certo, pois isso criaria um problema grave para ser resolvido pela administração, que é a falta de infraestrutura: conectividade deficiente, tomadas de energia inexistentes, e número insuficiente de projetores. Nesta mesma escola, foram encontrados tablets ainda dentro da embalagem original, mofados. 
Tabela 2 - Fatores influenciadores na implantação de tablets educacionais em larga escala. FATOR DEFINIÇÃO

Deve haver um planejamento envolvendo todos os outros fatores aqui listados. O

Planejamento planejamento, refletido em políticas operacionais, devem ser realizados projetos piloto em ambientes representativos da realidade antes da implantação em larga escala.

Deve haver mecanismos de auditoria e controle do uso dos dispositivos por parte dos

Auditoria professores, de modo a permitir a identificação e correção de falhas operacionais. Deve se estabelecer metas para obtenção de resultados monitorados do projeto.

Os interessados diretos e indiretos devem ser comunicados sobre a implantação,

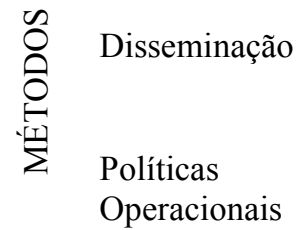
incluindo alunos e suas famílias, funcionários e professores, de modo que todos saibam como ela ocorrerá, que recursos serão disponibilizados e os resultados esperados. O planejamento deve contar com um conjunto de políticas operacionais que definam Operacionais vários dos processos envolvidos, como política de manutenção, produção de conteúdo e regras de uso dos tablets pelos servidores.

Um currículo apropriado com a nova realidade pedagógica propiciada pelo uso dos

Currículo tablets deve ser definida, de modo a permitir que as novas aulas podem ser concebidas para obter os melhores resultados de aprendizado com o uso dos tablets.

Novos métodos para uso das tecnologias envolvidas devem ser disseminados entre

Metodologia professores e pedagogos, de modo que tenham como projetas atividades educacionais adequadas à nova realidade de sala de aula.

Deve existir uma política bem definida de manutenção e substituição dos tablets

Manutenção durante toda a vida útil dos mesmos, reduzindo o tempo de espera do professor pelo dispositivo.

\begin{tabular}{|c|}
\hline Hardware \\
\hline Hardware \\
\hline
\end{tabular}

O hardware deve ter especificação compatível com a realidade da infraestrutura disponível, com memória que permite contornar a falta de conectividade, bateria de longa duração e desempenho adequado ao conteúdo.

Deve haver conectividade de Internet, de número de projetos/TV, de móveis e de tomadas elétricas em níveis compatíveis com a demanda criada pelo uso dos tablets. Deve existir um portal com recursos educacionais abertos compatíveis com os tablets

Conteúdo usados e com a conectividade disponível nas escolas. Os professores devem ser capacitados para adaptar estes recursos às suas necessidades e produzir seu próprio conteúdo.

Inclusão

Digital A implantação deve começar pelos professores com maior nível de inclusão digital, de modo que disseminem os conceitos, ideias e resultados obtidos pelo uso dos tablets, ao mesmo tempo que o restante tem tempo para elevar seu nível de inclusão digital.

Deve se estimular o surgimento de lideranças informais locais durante a implantação,

Liderança para que sirvam de referência para o uso dos tablets. Os líderes formais (gestores) devem ser cobrados por resultados em suas escolas.

Os envolvidos (gestores, professores, pedagogos e técnicos) devem ser capacitados

Capacitação não apenas no uso operacional, mas também e principalmente no uso pedagógico do tablet, como também na produção/adaptação de conteúdo e em direito autoral.

Cada escola deve contar, em período integral e com recursos humanos efetivos, com suporte técnico no uso operacional do tablet, bem como resolver problemas de natureza técnica e esclarecer dúvidas que envolvam o uso dos mesmos.

Suporte

Pedagógico

Cada escola, ou região escolar, deve contar com pessoal apto a dar suporte pedagógico aos professores, tanto na criação de conteúdo quanto no desenho metodológico das aulas.

O engajamento do professore é o resultado de todos os demais fatores, e deve ser

Engajamento estimulado com a adequada presença dos mesmos na escola. O professor deve ser estimulado a se engajar na implantação com incentivos cabíveis em cada instituição, do Professor mas a simples distribuição dos dispositivos sem estabelecimento de compromisso pelo professor deve ser evitada. 
A atitude dos professores também foi mencionada por diretores de escolas e pelo pessoal de suporte técnico e pedagógico, ao afirmarem que há professores que não tinham qualquer compromisso com o projeto, mesmo mediante condições apropriadas para tal.

Por isso o engajamento do professor, além de ser considerado consequência da adequada oferta de todos os outros fatores, também é um fator influenciador em si, pois a atitude de se engajar, ou não, à implantação também era uma questão de fórum íntimo. Alguns simplesmente não queriam sair de suas respectivas zonas de conforto, estimulados por um planejamento de implantação vago em aspectos que tinham que ser detalhados.

Tais constatações vão ao encontro com as críticas iniciais descritas neste artigo em relação à implantação dos dispositivos, e reforça o temor de que se esteja perdendo uma excelente oportunidade de aprimorar a educação pública com o uso de uma tecnologia que está presente na vida da grande maioria dos alunos de nossa sociedade. Por outro lado, este estudo explica pelo menos em parte o porquê de tais críticas encontrarem base na realidade da educação pública no Brasil, ao mesmo tempo que mapeia o que deve ser considerado para que uma implantação deste tipo venha a ser realizada.

\section{Conclusões}

O MILETE, ou Modelo de Implantação em Larga Escala de Tablets Educacionais, permite que se tenha uma percepção dos principais fatores que influenciam o tipo de implantação estudado e norteia gestores neste processo. Apesar disso implicar que os objetivos da pesquisa foram plenamente alcançados, não se tem a pretensão que este modelo possa ser generalizado a qualquer distrito escolar que venha a implantar estes dispositivos móveis em larga escala. Afinal, o que fundamentou o modelo foi dados oriundos da realidade de escolas brasileiras. Porém, como esta realidade segue padrões razoavelmente genéricos, pois conta com professores, sala de aula, alunos, conectividade, projetores, suporte técnico, entre outros elementos que se espera encontrar em qualquer escola, independente de cultura ou região, espera-se que sirva pelo menos de referência para análise inicial para planejamento da implantação.

\section{Referências Bibliográficas}

CARDOSO, C. Tablets na sala de aula: Mais do mesmo. Carta Capital, 07 jul. de 2012. Disponível em $<$ http://www.cartacapital.com.br/educacao/tablets-na-sabela-de-aula-maisdo-mesmo $>$ Acessado em 23 out. 2015.

CLARKE, B.; SVANAES, S. One-to-one tablets in secondary schools: An evaluation study. Tablets for Schools, dez. de 2012. Disponível em <http://www.medienberatung. schulministerium.nrw.de/Medienberatung-NRW/Lern-IT/Dokumente/Tablets/One-to-oneTablets-in-Secondary-Schools.pdf $>$ Acessado 23 out. 2015.

CORBIN, J.; STRAUSS, A. Basics of qualitative research: Techniques and procedures for developing grounded theory. Terceira ed. Thousand Oaks: Sage Publications. 2008, $379 \mathrm{p}$.

CRESWELL, J. W. Research Design Qualitative, Quantitative, and Mixed Methods Approaches. Terceira ed. Los Angeles: SAGE. 2009, 273 p.

DONALDSON, R. L. Student acceptance of mobile learning. Tallahassee: Florida State University, 2011, Tese de doutorado. 


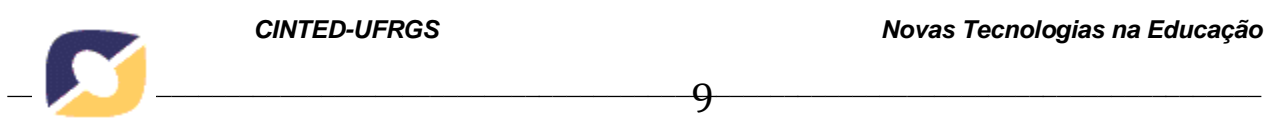

FARIAS, G.; ALLY, M. Large Scale Deployment of Tablet Computers in High-Schools: More than User's Adoption, Teaching Methods, and Learning Outcomes. In $\mathbf{3}^{\mathbf{0}}$

ICEDUTECH - International Conference on Educational Technologies, 2015, Florianópolis. Anais, Florianópolis: International Association for Development of Information Society, 2015.

FERREIRA, D. Inclusão digital na escola e a falta de estrutura. Professor Edgley Alexandre. São Paulo, 14 out. 2013. Disponível em <http://www.profedigleyalexandre.com/2013/10/inclusao-digital-na-escola-e-a-falta-de-estrutura.html> Acessado em 14 out. 2015.

INEP. Resultados e Metas do IDEB. Disponível em: < http://ideb.inep.gov.br/resultado/ resultado/resultadoBrasil.seam?cid=1334597> Acesso em 2 out. 2016.

KASTRENAKES, J. Los Angeles schools halt plan to give an iPad to every student. The Verge, 24 ago. 2014. Disponível em <http://www.theverge.com/2014/8/26/6069121/ los-angeles-schools-halt-plan-ipad-rollout $>$ Acessado em 14 out. 2015.

KWANG, K. Singapore SOE deployment plods on despite deadline. ZDNet, 24 set. 2012. Disponível em $<$ http://www.zdnet.com/article/singapore-soe-deployment-plods-on-despitedeadline-7000004704/> Acessado em 23 out. 2015

LIMA R. Tablet na escola. Revista Espaço Acadêmico, 31 ago. 2013. Disponível em $<$ http://espacoacademico.wordpress.com/2013/08/31/tablets-na-escola/> Acessado em 23 out. 2015.

LUCAS, L. Tablets em sala de aula: Análise das percepções dos professores de Matemática na escola pública de Taperoá/PB. Campina Grande: Universidade Estadual da Paraíba, 2014, Monografia de pós-graduação lato senso.

MCGEE, D.; VANDERNOOR, J. Tablet computer adoption programs in high schools. The effective technology plan, 6 jun. 2013. Disponível em $<$ http://280edtech.pbworks.com/w/ file/fetch/67603583/Tablet\%20Computing\%20in\%20High\%20School\%20Dean $\% 20$ and $\% 2$ 0Jeff\%206.24.13.docx> Acessado em 23 out. 2015.

MERCADANTE, A. (2013, July 16). Projeto Tablet Educacional: Entrevista com o Ministro da Educação. YouTube, 16 jul. 2013. Disponível em $<$ https://www.youtube.com/watch?v=wLyz0xUGcJ8> Acessado em 23 out. 2015.

NANDA, P.; AGARWAL, S. Government close to giving up on Aakash project. Live Mint e-News, 23 mar. 2013. Disponível em <http://www.livemint.com/Politics/fmEi8gsOSFg OzSTFfLsw6J/Govt-almost-gives-up-on-Aakash-says-no-point-in-hardware-ob.html> Acessado em 23 out. 2015.

NASCIMENTO, F. O uso pedagógico do tablet educacional como ferramenta de aprendizagem de alunos do primeiro ano regular do ensino médio. Campina Grande: Universidade Estadual da Paraíba, 2014, Monografia de pós-graduação lato senso..

NEVES, A.; CARDOSO, C. Os desafios do uso do tablet pelos professores do Ensino Médio das escolas públicas do Distrito Federal. In $5^{0}$ Simpósio Hipertexto e Tecnologias na Educação, 2013, Recife. Anais, Recife: Núcleo de Estudos de Hipertexto e Tecnologia Educacional, 2013. 
OECD. What 15-year-olds know and what they can do with what they know. PISA 2012 Results in Focus, Paris, 2012. 44p. Disponível em $<$ http://www.oecd.org/pisa/keyfindings /pisa-2012-results-overview.pdf $>$ Acessado em 23 out. 2015.

OLIVEIRA, D. Percepção dos docentes sobre o uso dos tablets na escola. Campina Grande: Universidade Estadual da Paraíba, 2016, Monografia de pós-graduação lato senso..

QUARESMA, C.; ABEGG, I.; GARCÊS, S.; FELIX, R. Tecnologias na educação: Inclusão digital dos professores da rede estadual a partir da implementação do programa Tablet Educacional. Revista Novas Tecnologias na Educação - Renote, Porto Alegre, v.12, n.1, 2014.

RODRIGUES, J. Apropriação didática do tablet educacional por docentes do ensino médio da Secretaria de Estado de Educação do Distrito Federal. Brasília: Universidade de Brasília, 2016, Dissertação de Mestrado.

SAKAWEE, S. Thailand's One Tablet Per Child faces another dead end as a Chinese manufacturer cancels the deal. Tachinasia, 3 fev. 2014. Disponível em $<$ https:/www.techinasia.com/thailands-tablet-child-faces-dead-chinese-manufacturercancels-deal/> Acessado em 23 out. 2015.

SANTANA, B.; ROSSINE, C.; PRETTO, N. Recursos educacionais abertos: Práticas colaborativas e políticas públicas. Salvador: Edufba, 2012. 249 p.

SOMMERRICH, C. M.; WARD, R.; SILKDAR, K.; PAYNE, J.; HERMAN, L. A survey of high school students with ubiquitous access to tablet PCs. Ergonomics, v.50, n.5, 2007.

SOUZA, L. Os desafios da utilização dos tablets em uma escola do ensino médio de Aparecida/PB. Campina Grande: Universidade Estadual da Paraíba, 2014, Monografia de pós-graduação lato senso..

TELES, G. Governo federal distribuirá 600 mil tablets para escolas públicas. Jornal da Globo, 2 fev. 2012. Disponível em <http://g1.globo.com/jornal-da-globo/noticia/2012/02 /governo-federal-distribuira-600-mil-tablets-para-escolas-publicas.html> Acessado em 23 out. 2015.

TERRA. País supera meta no IDEB, mas ensino médio piora em 9 Estados. Terra Magazine, 14 ago. 2012. Disponível em http://noticias.terra.com.br/educacao/pais-superameta-no-ideb-mas-ensino-medio-piora-em-9-estados,50bb42ba7d2da310V gnCLD200000 bbcceb0aRCRD.html> Acessado em 23 out. 2015.

TRONT, J. G. Practices through a large-scale tablet personal computer deployment. IEEE Computer Society, v.40, n. 9, 2007.

VALSTAD, H. (2011). Introducing the iPad in a Norwegian high school: How do students and teachers react to this technology. Oslo: Norwegian University of Science and Technology, 2011. Dissertação de Mestrado.

WANG, Y.; HUANG, C. The Effect of Visual Cueing and Control Design on Children's Reading Achievement of Audio E-books with Tablet Computers. Journal of Curriculum and Teaching, v.4, n. 1, 2015. 


\section{Agradecimento}

Os autores deste artigo agradecem à agência de fomento de pesquisa Alberta Innovates, do Governo Provincial de Alberta, Canadá, que custeou toda esta pesquisa e as despesas de estudo de doutoramento a ela associadas com o prêmio Technology Futures. 